\title{
THE DUST RING OF LUMINOUS BLUE VARIABLE CANDIDATE HD 168625: INFRARED OBSERVATIONS AND MODEL CALCULATIONS ${ }^{1}$
}

\author{
Timothy B. O’Hara, ${ }^{2}$ Margaret Meixner, ${ }^{3}$ Angela K. Speck, ${ }^{4}$ and Toshiya Ueta ${ }^{5}$ \\ Department of Astronomy, University of Illinois at Urbana-Champaign, 1002 West Green Street, Urbana, IL 61801; \\ tbohara@uiuc.edu,meixner@stsci.edu,speckan@missouri.edu, ueta@oma.be \\ AND \\ MATTHEW BoBRowsKy \\ Challenger Center for Space Science Education, 1250 North Pitt Street, Alexandria, VA 22314; \\ mbobrowsky@challenger.org \\ Received 2003 January 22; accepted 2003 August 11
}

\begin{abstract}
We present a $2.218 \mu \mathrm{m}$ image from the Hubble Space Telescope/Near Infrared Camera and Multi-Object Spectrometer (NICMOS) and a $55 \mu \mathrm{m}$ image from ISOPHOT of the dust ring surrounding the luminous blue variable (LBV) candidate HD 168625, together with new temperature and optical depth maps derived from mid-IR images. The shell is detached from the star in the near-IR, and substructure in the overall toroidal shell is visible. The far-IR image constrains the extent of the dust shell to $\sim 25^{\prime \prime}$ in diameter, providing an upper radius limit for modeling. The temperature maps and the NICMOS image show evidence for very small transiently heated dust grains in the shell. The opacity maps show higher optical depth in the limbs, consistent with interpretation of the dust shell as an equatorially enhanced torus inclined $\sim 60^{\circ}$ with respect to the observer. An overall trend in the dust emission location with wavelength is observed and interpreted as a variation with respect to location in the nebula of either the dust grain size distribution or gas-to-dust mass ratio. Radiative transfer calculations using 2-Dust indicate that a mass-loss event occurred $\sim 5700 \mathrm{yr}$ ago with a rate of $(1.9 \pm 0.1) \times 10^{-4} M_{\odot} \mathrm{yr}^{-1}$, creating a dust torus that currently has a $\tau_{V} \sim 0.22$ in the equatorial plane and a dust mass of $(2.5 \pm 0.1) \times 10^{-3} M_{\odot}$. Using published values for the gas mass, we find a gas-to-dust mass ratio of 840 , which is $\sim 4$ times higher than current estimates for the interstellar medium. In addition to a high equator-to-pole density ratio $(\sim 31)$ torus, an elliptical midshell is needed to reproduce the appearance and spectral energy distribution of the dust. Therefore, HD 168625 is an excellent example of proposed models of LBV nebulae in which a stellar wind interacts with a preexisting density contrast and creates a blowout in the polar direction perpendicular to the equatorial ring. The circumstellar shell is much lower in mass than that of LBV $\eta$ Carinae, suggesting that HD 168625 had a lower mass progenitor.

Subject headings: circumstellar matter — infrared: stars — stars: individual (HD 168625) —
\end{abstract} stars: mass loss — stars: variables: other

\section{INTRODUCTION}

The designation of luminous blue variable (LBV) was first suggested by Conti (1984) and clarified by Humphreys \& Davidson (1994). LBVs feature microvariability of up to a few tenths of a magnitude over timescales of a few weeks or months and larger, irregular photometric variability with amplitudes of 1-2 mag on timescales of years or decades, indicating, perhaps, a sudden increase in mass-loss rate (Davidson 1987) or changes in the radius of the star

\footnotetext{
${ }^{1}$ Based in part on observations made with the NASA/ESA Hubble Space Telescope, obtained at the Space Telescope Science Institute, which is operated by the Association of Universities for Research in Astronomy, Inc., under NASA contract NAS5-26555; these observations are associated with proposal 7898. Based also on observations with ISO, an ESA project with instruments funded by ESA member states (especially the PI countries: France, Germany, the Netherlands, and the United Kingdom) and with the participation of ISAS and NASA.

${ }^{2}$ Also at Department of Physics, University of Illinois at UrbanaChampaign, 1110 West Green Street, Urbana, IL 61801.

${ }^{3}$ Current address: Space Telescope Science Institute, 3700 San Martin Drive, Baltimore, MD 21218.

${ }^{4}$ Current address: Department of Physics and Astronomy, 223 Physics Building, University of Missouri, Columbia, MO 65211.

${ }^{5}$ Current address: Royal Observatory of Belgium, Ringlaan 3, B-1180 Brussels, Belgium.
}

(Leitherer et al. 1989). In addition, some LBVs exhibit major outbursts between prolonged periods of quiescence, the most famous examples being $\mathrm{P}$ Cygni and $\eta$ Carinae. The temperature of LBVs likewise varies, from a maximum during quiescence of $T_{\text {eff }}>15,000 \mathrm{~K}$ to a minimum of $T_{\text {eff }} \sim 8000 \mathrm{~K}$ during periods of mass loss.

Although observation of the actual eruption is rare, the presence of optical nebulae around confirmed or candidate LBVs implies that such outbursts are a common aspect of LBV behavior (Nota et al. 1995). For example, ring nebulae around LBVs AG Car, HR Car, and R127 have each been found to contain several solar masses of material, presumably shed in $\eta$ Car-like eruptions (Nota et al. 1992; Clampin et al. 1993, 1995).

HD 168625 (IRAS 18184-1623) was first identified as a candidate LBV by Chentsov \& Luud (1989), who classified it as spectral type B5.6 \pm 0.3 with a $T_{\text {eff }} \simeq 13,000 \mathrm{~K}$. Nota et al. (1996) have observed variation in its effective temperature of $\simeq 13,000-15,000 \mathrm{~K}$ and a P Cygni profile that indicates a stellar wind with a velocity of $183 \mathrm{~km} \mathrm{~s}^{-1}$ and a massloss rate $\geq(1.2 \pm 0.2) \times 10^{-6} M_{\odot} \mathrm{yr}^{-1}$. The distance to HD 168625 was estimated by van Genderen et al. (1992), who claimed a distance of $2.2 \mathrm{kpc}$ based on association with HD 168607 and M17 due to angular proximity. This association has been challenged, however, on the basis of systemic 
velocity measurements. Robberto \& Herbst (1998) used infrared observations and estimates of stellar parameters to obtain a distance of $1.2 \mathrm{kpc}$. Most recently, Pasquali et al. (2002) derived a kinematic distance of $2.8 \mathrm{kpc}$, which we believe to be more reliable and adopt here.

The timescale of HD 168625's light variability was measured by van Genderen et al. (1992), who found a quasi-period of 35 days, which they did not consider unusual enough to grant LBV status to this object. However, the nebula around HD 168625 was determined to be LBV-like, with an ellipsoidal shell expanding at $\sim 20$ $\mathrm{km} \mathrm{s}^{-1}$, by Hutsemékers et al. (1994) on the basis of near-infrared and visible imaging and spectroscopy. With further observations Nota et al. (1996) concluded that HD 168625 has not shown variability typical of LBVs in the last few decades but that its nebula indicated an LBV-like major outburst $\sim 10^{3} \mathrm{yr}$ ago. The question of whether HD 168625 is, in fact, an LBV is not yet settled, and given the rather loose definition of LBVs the question may never be answered to everyone's satisfaction. We do not enter into this debate here, and we refer to HD 168625 as an LBV in this paper.

Since its discovery by Hutsemékers et al. (1994) and its detailed study by Nota et al. (1996), the nebula of HD 168625 has been the focus of three additional studies. As part of a larger mid-infrared imaging survey of 78 protoplanetary nebula (PPN) candidates, Meixner et al. (1999) imaged HD 168625 at 8.8, 12.5, and $20.6 \mu \mathrm{m}$, revealing a toroidal dust shell more spectacular than any of the PPN sources, but performed no quantitative analysis of the dust shell. Based on their mid-infrared images at 4.7, 10.1, 11.6, and $19.9 \mu \mathrm{m}$, Robberto \& Herbst (1998) derived a dust temperature of $135 \mathrm{~K}$ and used an analytic spherical model to estimate an optical depth of $\tau_{V} \sim 0.2$ and a dust mass of $2.8 \times 10^{-3} M_{\odot}$. Robberto \& Herbst (1998) also suggested that the nebular material was a swept up red supergiant wind, that HD 168625 was evolving to hotter temperatures, and that a larger neutral envelope extended beyond the dust shell observed in the mid-IR. A multiwavelength study by Pasquali et al. (2002) found a dust temperature of $113 \mathrm{~K}$ based on ISOCAM data, an ionized gas mass of $2.1 M_{\odot}$ based on $\mathrm{H} \alpha$ and continuum Hubble Space Telescope (HST)/ WFPC2 images, and a nebular expansion velocity of 19 $\mathrm{km} \mathrm{s}^{-1}$ based on ground-based echelle spectra. The HST/WFPC2 images clearly show the bipolar morphology. However, none of these studies has quantitatively derived geometric parameters for the dust shell morphology, nor have detailed radiative transfer calculations of the dust shell been performed.

Here we present a high-resolution near-infrared image of HD 168625 from the Near Infrared Camera and MultiObject Spectrometer (NICMOS) on HST and a far-infrared image from ISOPHOT on the Infrared Space Observatory $(I S O)$ in $\S 2$. We derive new temperature and opacity maps from the Meixner et al. (1999) mid-infrared imaging in $\S 3$, and their implications for dust shell structure are discussed. The qualitative picture of HD 168625 created from these data is discussed in $\S 4$. In $\S 5$ we present detailed radiative transfer models using the axisymmetric code 2-Dust and discuss the quantitatively derived geometry parameters for the dust shell and the inferred history of mass loss. In $\S 6$ we discuss how HD 168625 compares with axisymmetric massloss parameters for LBVs and with lower mass PPNs.
Finally, in $\S 7$ we combine our overall qualitative and quantitative results.

\section{OBSERVATIONS AND RESULTS}

\subsection{Near-IR Imaging}

Observations with NICMOS on HST were obtained on 1998 August 31. Exposures were taken at four positions centered around $18^{\mathrm{h}} 21^{\mathrm{m}} 20^{\mathrm{s}}-16^{\circ} 22^{\prime} 28^{\prime \prime}$ using camera 2 (NIC2), which has a field size of 19 ". $2 \times 19$ !'2 at a pixel scale of 0 ! 075 . All images were taken using filter F222M, which has central wavelength $2.218 \mu \mathrm{m}$ and FWHM $0.145 \mu \mathrm{m}$.

Data were taken using the MULTIACCUM readout mode, which nondestructively reads counts at intermediate exposure times, facilitating removal of cosmic-ray hits and saturated pixels. Exposure time was 192 s, using the predefined sampling sequence STEP32, which takes rapid exposures up to $32 \mathrm{~s}$ (at 0.3, 0.6, 1.0, 2.0, 4.0, 8.0, 16.0, and 32.0 s) and every $32.0 \mathrm{~s}$ thereafter. The four exposures were taken in a spiral dither pattern with steps of $\sim 9^{\prime \prime}$ between images.

The data were recalibrated using the STSDAS calnica task in IRAF (Dickinson et al. 2002), with updated nonlinearity and temperature-dependent synthetic flat-field reference files. The CRIDCALC task was used to identify possible cosmic-ray hits by checking for nonlinear count rate increases between readouts of the MULTIACCUM sequence.

The standard bad-pixel mask provided by STScI was used to flag permanently hot and cold pixels for exclusion during the mosaicking process. In addition, images were checked against a mask of known pixels of reduced sensitivity ("grot"), and pixels with visually obvious abnormally increased or decreased signal were also flagged. Permanently bad pixels and grot that fell outside overlapping areas of images, and thus would not be dithered over in the mosaicking process, were filled in by nearest neighbor interpolation. This process was reasonable since the scale of structures in the target object is much larger than 1 pixel in extent.

The four individual exposures were combined using the STSDAS task calnicb, which estimates and removes the background signal from each image, calculates the relative positions of the images from observation logs, and constructs a mosaic image using error-weighted means of all samples in regions of overlapping images, exclusive of bad pixels flagged during previous calibration steps.

NIC2 includes a coronagraphic hole in one quadrant with diameter $\sim 1^{\prime \prime}$. In two of the four images the region containing the hole was overlapped by another image, and the hole was removed in the mosaicking process. In the other two cases the hole remains in the final mosaic image as a region of zero signal because the area the hole covers is too large to reasonably interpolate over.

The NICMOS image is shown in Figure 1a. Because of the obvious saturation of the star, it is impossible to obtain a reasonable estimate of the total flux at this wavelength. The dust shell is clearly detached from the star in the south and west. At this resolution inhomogeneities in the dust shell are visible; in particular, the southern rim appears to be a fragmented arc. Such fragmentation is commonly seen in LBV nebulae and is probably created when a fast wind interacts with the massloss eruption (Nota et al. 1995). 


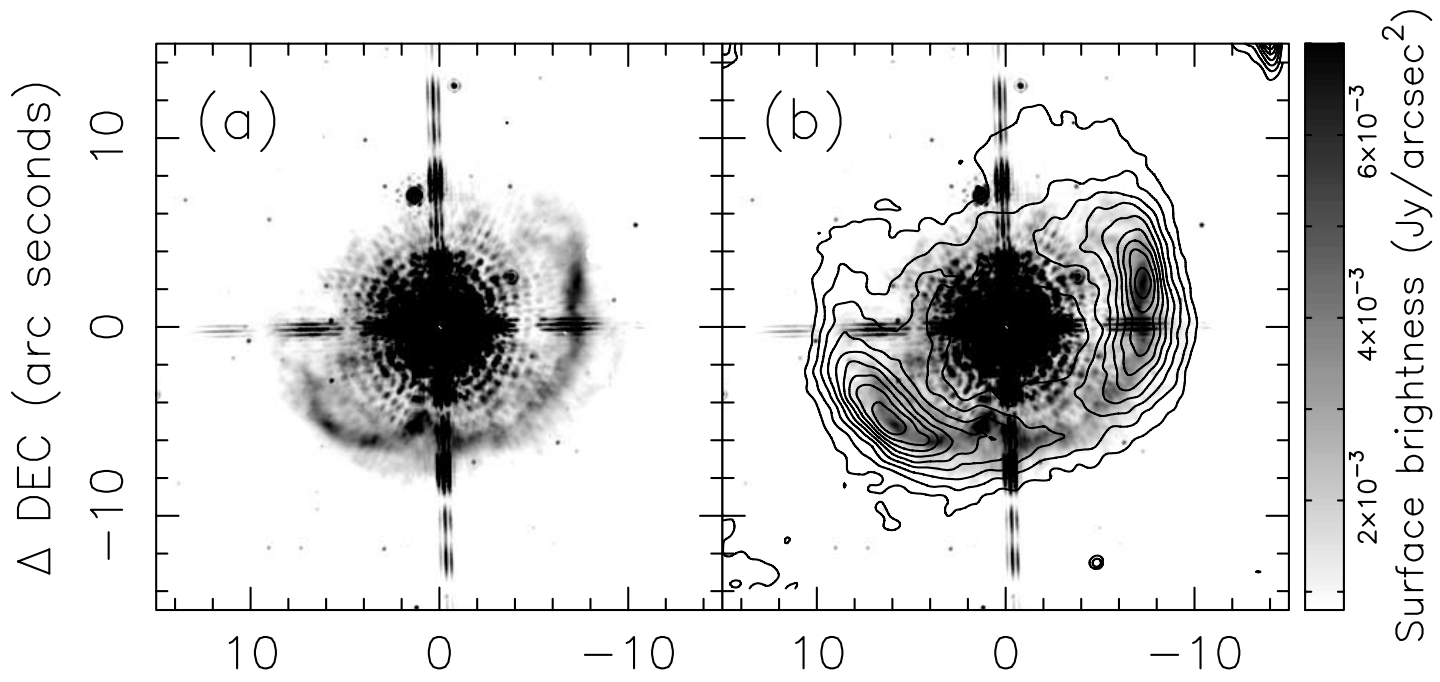

$\triangle$ RA (arc seconds)

FIG. 1. - (a) NICMOS image at $2.2 \mu \mathrm{m}$ with north up and east to the left. The star itself is saturated in this image. The coronagraphic hole appears roughly $8^{\prime \prime}$ west and $4^{\prime \prime}$ north of center. (b) Same NICMOS image with the $12.5 \mu \mathrm{m}$ image of Meixner et al. (1999) contoured on top. Contours begin at $10 \%$ of peak intensity $\left(0.4 \mathrm{Jy} \operatorname{arcsec}^{-2}\right)$ and are spaced at intervals of $10 \%$.

For comparison, the NICMOS image is shown overlaid on the $12.5 \mu \mathrm{m}$ image of Meixner et al. (1999) in Figure $1 b$. The spatial extent of the nebula is roughly the same at these wavelengths. This spatial coincidence indicates that the shell seen in the NICMOS image is due to dust emission. Indeed, the $K$-band spectrum of HD 168625 (Hanson, Conti, \& Rieke 1996 1996) suggests that there are no emission lines in the $2-2.2 \mu \mathrm{m}$ band along the line of sight to the central star. Based on comparison of narrowband and broadband images in the $2-2.2 \mu \mathrm{m}$ wavelength region, Hutsemékers et al. (1994) similarly suggest that the $K$-band image is continuum dominated.

Because of the size of the dust shell (inner radius $\sim 0.1 \mathrm{pc}$ ), it is not possible for the grains to be in thermal equilibrium at a temperature high enough for equilibrium emission in the near-IR. Indeed, a mid-IR spectrum of HD 168625 (Skinner 1997) reveals polycyclic aromatic hydrocarbon (PAH) features and suggests that nonequilibrium grain emission also affects the 8.8 and $12.5 \mu \mathrm{m}$ image of Meixner et al. (1999).

\subsection{Far-IR Imaging}

Far-infrared observations of HD 168625 were taken on 1997 April 12 using the ISO (Kessler et al. 1996). We obtained a far-IR image using the imaging spectrophotopolarimeter on ISO (ISOPHOT; Lemke et al. 1996). The image is centered on source at $18^{\mathrm{h}} 21^{\mathrm{m}} 19^{\mathrm{s}}-16^{\circ} 22^{\prime} 26^{\prime \prime}$. (J2000). The position angle of the image was determined by the spacecraft orientation at 4.4 east of north.

The image was taken using the C100 $50 \mu \mathrm{m}$ filter, which has a $3 \times 3$ pixel format with $46^{\prime \prime}$ pixel scale. Each position was imaged twice in order to determine the repeatability of structures observed in the object's spatial profile. The PHT32 AOT uses a combination of raster mapping and chopper sweeping to create a map. There were eight raster positions with raster length of $8^{\prime}$, at steps of $60^{\prime \prime}$; however, the chopper was used to make smaller steps of $15^{\prime \prime}$. There were four lines on rastering with $15^{\prime \prime}$ between adjacent lines. This gives an image pixel size of $15^{\prime \prime} \times 15^{\prime \prime}$. The point-spread function (PSF) has FWHM of 48".5.

The ISOPHOT data presented in this paper were reduced using the PHOT Interactive Analysis (PIA) package (Gabriel et al. 1997), together with the P32Tools package developed specifically for reduction of the AOT PHT32 data (Schulz \& Peschke 2002). The P32Tools package allows a better calibration of the PHT32 data, so that the errors on the calibrated data can be reduced to $\sim 30 \%$.

This analysis provided an estimate of a total flux of $40 \pm 12 \mathrm{Jy}$ at $55 \mu \mathrm{m}$, in contrast to the $60 \mu \mathrm{m} I R A S$ flux of $117 \mathrm{Jy}$ with similar filter characteristics. The IRAS data is assessed as "moderate quality"; furthermore, HD 168625 is located $15^{\prime}$ southeast of M17, one of the most massive star formation regions in the galaxy, which leads to a high level of confusion for the far-IR IRAS data. Since the ISO beam is smaller than the IRAS beam, it is better able to separate the source from the background and is a more reliable flux. Hence, for our modeling work we have placed more emphasis on the ISO flux value and treat the IRAS flux as an upper bound.

We have simulated the $55 \mu \mathrm{m}$ image using a mapping simulator as part of the PIA package (Gabriel \& Hur 2000). This simulator allows us to build a piece of sky using point and/or extended sources with or without a gradient/varying background. The simulator then convolves this sky with a PSF, accounting for the particular raster pattern used in observations, to produce an image showing how this object would appear if observed using the PHT32 AOT and assuming a certain noise level.

In Figure 2 the observed emission (Fig. $2 a$ ) is compared with simulated emission (Fig. 2b). We find that in order to match the observed image, the source must be a point source on a gradient background. Since the source in the data image appears to be effectively a point source for this instrument, and given the PSF width of 48 " 5 , we conclude that 


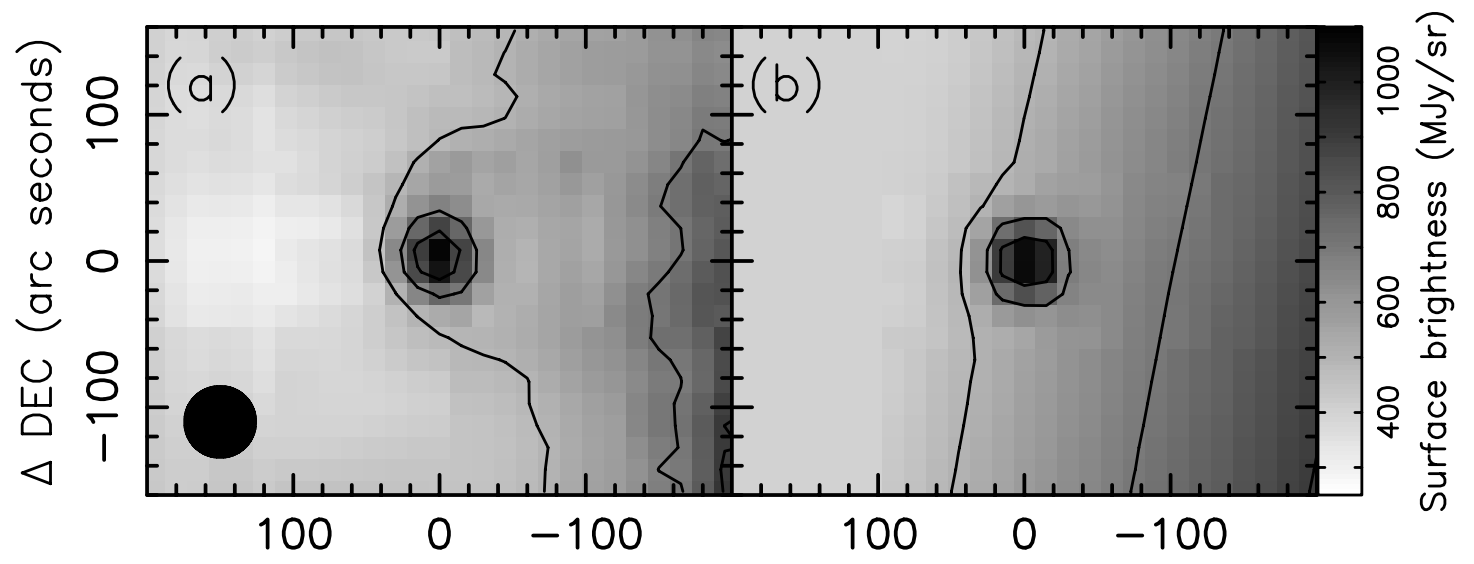

$\triangle$ RA (arc seconds)

FIG. 2.- (a) ISO image at $55 \mu \mathrm{m}$ and (b) simulation image of a point source for the same camera, with north up and east to the left. Base contour is $500 \mathrm{MJy} \mathrm{sr}^{-1}$, and contours are spaced at intervals of $200 \mathrm{MJy} \mathrm{sr}^{-1}$. The PSF (48".5) is shown on the lower left in the data image.

the dust shell is $\leq 25^{\prime \prime}$ in diameter, comparable to its size in the near- and mid-IR.

\section{DUST TEMPERATURE AND OPACITY MAPS}

In order to discern the color temperature and opacity of the circumstellar dust shell, we used a semianalytic approach to combine the $8.8,12.5$, and $20.6 \mu \mathrm{m}$ midinfrared images of HD 168625 obtained by Meixner et al. (1999), following the method of Dayal et al. (1998). Two sets of maps were created: (1) a temperature map and an optical depth map at $12.5 \mu \mathrm{m}$ using the 8.8 and $12.5 \mu \mathrm{m}$ images and (2) a temperature map and an optical depth map at $20.6 \mu \mathrm{m}$ using the 12.5 and $20.6 \mu \mathrm{m}$ images. The images were centered relative to one another by comparing the locations of the limb brightness peaks, and the $12.5 \mu \mathrm{m}$ image was convolved with a Gaussian of appropriate size to match the resolution of the 8.8 and $20.6 \mu \mathrm{m}$ images.

Figure 3 shows dramatic differences in the two sets. The first (from the 8.8 and $12.5 \mu \mathrm{m}$ images) predicts optical depths at $12.5 \mu \mathrm{m}$ between $10^{-4}$ and $10^{-3}$ (Fig. $3 a$ ), in rough agreement with the model results given below. However, it also implies dust shell temperatures up to several hundred degrees (Fig. 3b), well above the equilibrium temperature estimates of Pasquali et al. (2002; $113 \mathrm{~K})$ and Robberto \& Herbst $(1998 ; 135 \mathrm{~K})$, as well as those of our modeling. In the second set of maps (derived from the 12.5 and $20.6 \mu \mathrm{m}$ images), a much higher optical depth of $0.1-1$ at $20.6 \mu \mathrm{m}$ is predicted (Fig. 3c), which is too high to match the mid-IR observations. The temperature of $\sim 120 \mathrm{~K}$ predicted by this set of maps, however (Fig. 4d), is much more in line with prior estimates and our modeling work.

The obvious inaccuracies of these parameter maps are almost certainly due in large part to the fact that the method used to create them assumes that emission is purely thermal and that the dust shell is at thermal equilibrium. As noted previously, much of the 8.8 and $12.5 \mu \mathrm{m}$ emission arises from PAHs. Therefore, using images at these wavelengths to derive temperature and opacity maps has some limitations. The derivation of these maps relies on an assumption of thermal equilibrium, but PAH emission arises from transient, nonequilibrium grain emission. As we cannot legiti- mately claim accuracy in derived optical depth from one set of maps and in temperature from the other, we do not attempt to draw quantitative conclusions from them.

Nevertheless, the structure of the optical depth maps and the temperature maps of the two sets is quite similar and indicates reliable, relative values of optical depth and temperature. The optical depth maps show the highest values on the northwest and southeast limbs, coincident with the peaks of mid-IR emission and suggesting the presence of a limb-brightened, nearly edge-on torus of dust. Comparing the temperature and opacity maps, we find that they are anticorrelated on the northern and southern rims. The temperature maps show a higher temperature on the southern edge of the dust shell, while the opacity maps show a higher optical depth on the northern edge. We discuss this anticorrelation in the context of other observations in the next section.

\section{THE QUALITATIVE PICTURE OF HD 168625}

The infrared dust emission from near-IR to far-IR is spatially coincident, indicating that all the dust resides in a geometrically thin, axisymmetric shell. The mid-IR optical depth maps suggest that this shell has limb-brightened peaks and is really a nearly edge-on torus. Our near-IR image most clearly shows the detachment of the dust torus from the star and reveals a clumpiness on small scales. This clumpiness may be caused by a nonhomogenous mass loss or by fragmentation of the shell when a fast wind plows into the slower wind. Optical continuum and $\mathrm{H} \alpha$ emission images suggest that a lower density elliptical bubble exists perpendicular to the dusty torus (Pasquali et al. 2002). Integrating all these observations, we arrive at a qualitative picture of HD 168625, shown schematically in Figure 4. The circumstellar shell includes both an optically thin equatorial torus and an even thinner elliptical or bipolar midshell along the polar axis.

Superposed on this global structure of the dust shell, we observe an interesting trend in the IR emissions. At the shortest wavelengths, the near-IR emission is brightest in the southern rim of the shell and there is a gap in the north. As the wavelength increases, the IR emission appears to 

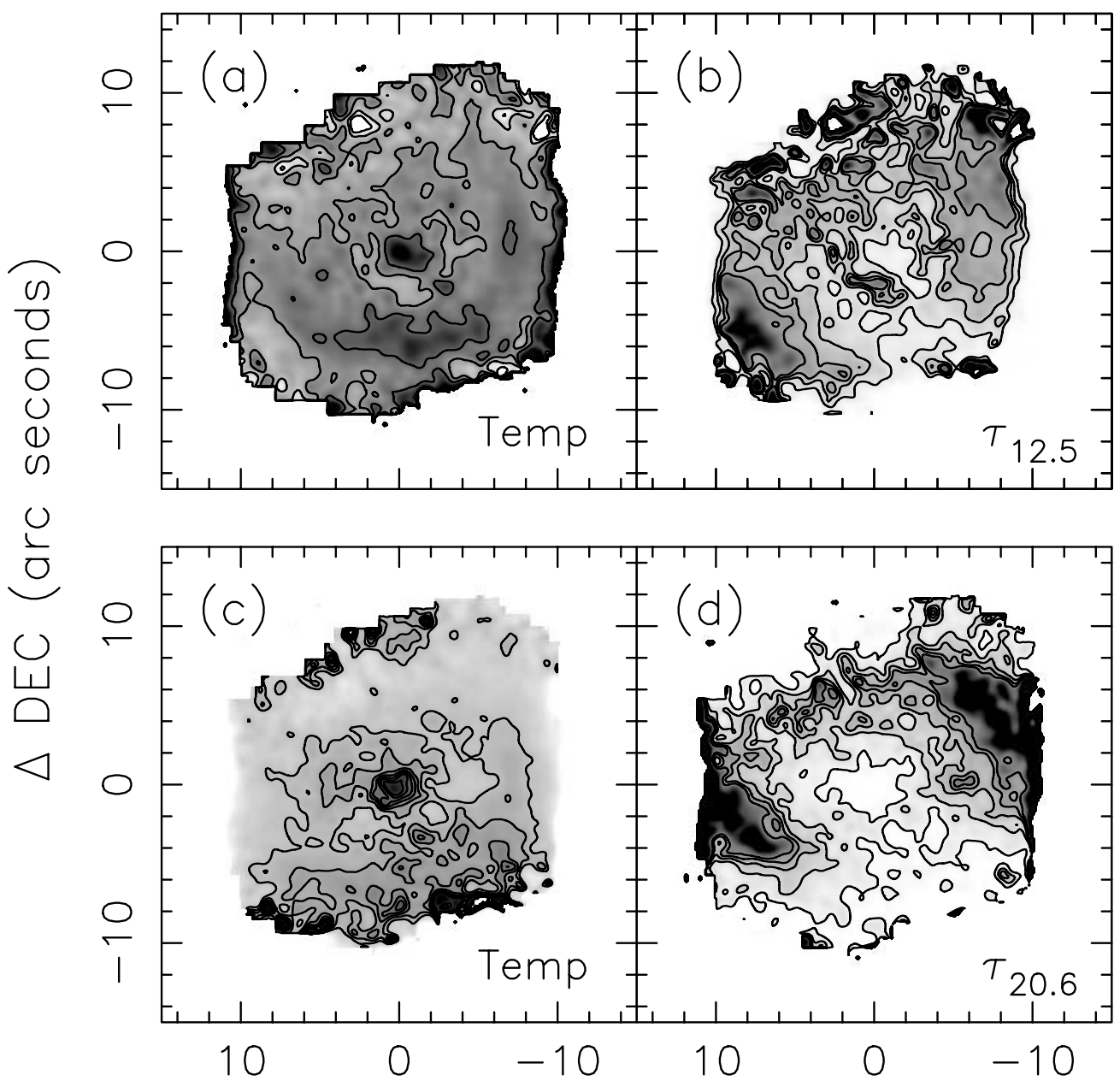

\section{$\triangle$ RA (arc seconds)}

FIG. 3.-Temperature map and optical depth map at $12.5 \mu \mathrm{m}$ derived from (a) $8.8 \mu \mathrm{m}$ and $(b) 12.5 \mu \mathrm{m}$ images, and temperature map and optical depth map at $20.6 \mu \mathrm{m}$ derived from $(c) 12.5 \mu \mathrm{m}$ and $(d) 20.6 \mu \mathrm{m}$ images, with north up and east to the left. In all images artifacts create spurious data around the edges. (a) Contours begin at $100 \mathrm{~K}$ and are spaced at intervals of $100 \mathrm{~K}$. (b) Contours begin at 0.0002 and are spaced at intervals of 0.0002 . (c) Contours begin at $110 \mathrm{~K}$ and are spaced at intervals of $10 \mathrm{~K} .(d)$ Contours begin at 0.1 and are spaced at intervals of 0.2 .

creep north until at $20.6 \mu \mathrm{m}$ the emission is brighter in the northern rim. A similar contrast has been observed in the optical by Pasquali et al. (2002). The optical reflection nebula is greatly enhanced on the northern rim of the dust shell, while the $\mathrm{H} \alpha$ emission appears much brighter on the southern rim. Pasquali et al. (2002) interpret these distinctive optical morphologies as a variation in the gas-to-dust mass ratio with respect to position in the nebula; it is highest in the south. Our optical depth maps certainly support their idea because the maps show a general increase in dust optical depth as position in the shell moves from south to north. However, these optical depth maps really trace the optical depth of larger grains and are not sensitive to the very small grains that are responsible for the near-IR emission. In fact, the dust temperature shows the opposite trend to the optical depth: it decreases from south to north and is essentially constant in the northern part of the shell. Smaller dust grains tend to get hotter than larger dust grains. Thus, an alternative explanation to this trend in IR emission may be that the grain size distribution varies with respect to position in the nebula. The smallest grains exist in the southern shell, and the grains become progressively larger toward the north. Dust grain shattering may result from a fast wind interacting with a preexisting dust shell, and the variation across the dust shell may indicate a preferred southern direction for the fast wind.

Our qualitative picture of HD 168625 supports the ideas proposed by Nota et al. (1995) and Dwarkadas \& Owocki (2002) for the formation of bipolar morphology observed in LBVs. HD 168625 has a fast wind with $v=183 \mathrm{~km} \mathrm{~s}^{-1}$ and $\dot{M} \geq(1.2 \pm 0.2) \times 10^{-6} M_{\odot} \mathrm{yr}^{-1}$ (Nota et al. 1996). The fast wind is interacting with the gas and dust shell ejected during the LBV outburst that is moving at a slower velocity, $19 \mathrm{~km} \mathrm{~s}^{-1}$ (Pasquali et al. 2002). This preexisting shell has an equatorial density enhancement, as indicated by the limb-brightened shell. The interaction of the winds produces a bubble that bursts out in a direction perpendicular to the torus, which we observe as an elliptical midshell. In the 


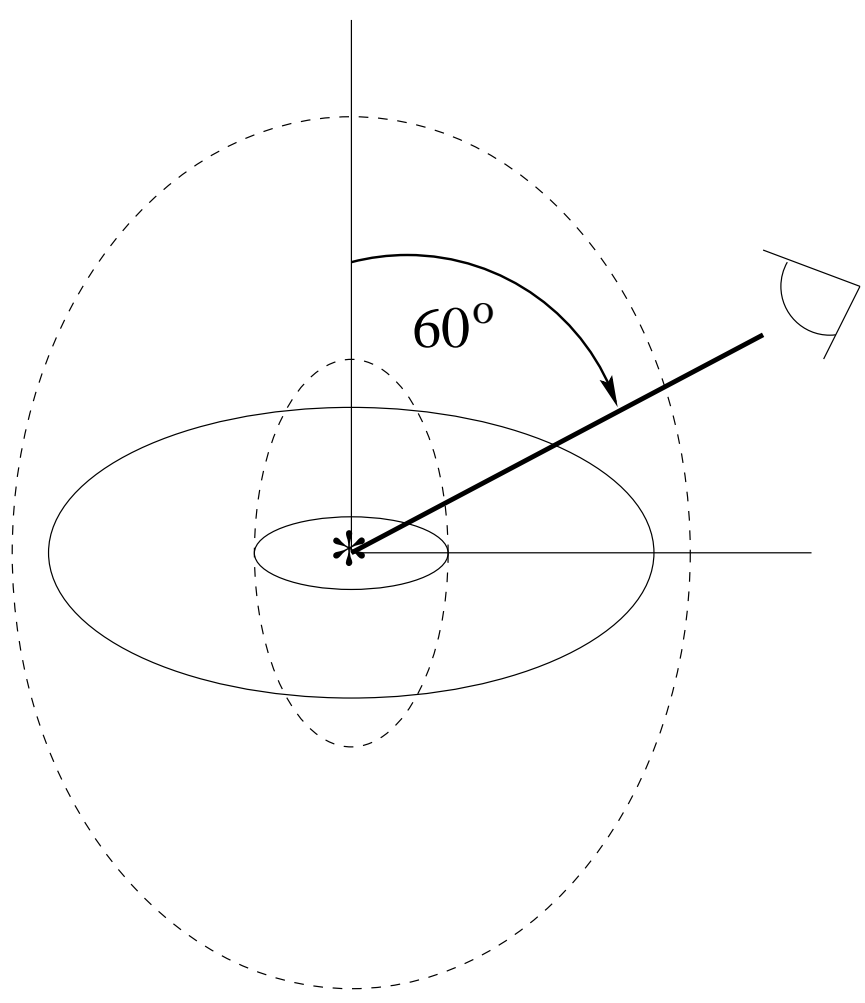

FIG. 4.-Schematic qualitative picture of HD 168625, as explained in the text. The dust shell consists primarily of a toroidal dust shell (solid line) but with an elliptical or bipolar midshell (dashed line) oriented along the symmetry axis.

next section, we derive a quantitative model for this qualitative picture.

\section{RADIATIVE TRANSFER MODELING}

\subsection{2-Dust Background}

In order to refine stellar parameters and dust shell morphology, we have modeled HD 168625 using the radiative transfer code 2-Dust (Ueta \& Meixner 2003). Our dust distribution has the form of an equatorial torus, an elliptical midshell, and, at the very outer radius, a spherical shell. This geometry is expressed analytically as

$$
\begin{aligned}
\rho(r, \theta)= & \rho_{\min }\left(\frac{r}{r_{\min }}\right)^{-B\left(1+C \sin ^{F} \theta\left\{\exp \left[-\left(r / r_{\mathrm{sw}}\right)^{D}\right] / \exp \left[-\left(r_{\min } / r_{\mathrm{sw}}\right)^{D}\right]\right\}\right)} \\
& \times\left[1+A(1-\cos \theta)^{F}\right. \\
& \left.\times\left\{\exp \left[-\left(r / r_{\mathrm{sw}}\right)^{E}\right] / \exp \left[-\left(r_{\min } / r_{\mathrm{sw}}\right)^{E}\right]\right\}\right]
\end{aligned}
$$

where $r$ is the radius within the limits of $r_{\min }$ and $r_{\max }, \theta$ is the latitude, and $\rho_{\min }$ is the dust mass density on the equatorial axis at the inner edge of the envelope.

The grain size distribution was modeled using a power law (Mathis, Rumpl, \& Nordsieck 1977):

$$
n(a)=a^{-3.5}, \quad a_{\min }<a<a_{\max },
$$

where the minimum grain size $a_{\min }$ and maximum grain size $a_{\max }$ are specified as inputs.

Modeling with 2-DusT produces both model images for comparison with observed images, and a model spectral energy distribution (SED), which can be compared to data after accounting for interstellar reddening.
An extensive discussion of the geometric parameters in equation (1) is given in Ueta \& Meixner (2003). For more examples of the use of 2-Dust and its earlier versions, see, e.g., Ueta et al. (2001a, 2001b) and Meixner et al. (2002).

\subsection{Constraints and Input Parameters}

In seeking a best-fit model for HD 168625 we were guided by comparisons to the SED from photometry and the IRAS Low-Resolution Spectrometer (LRS), as well as comparisons to infrared imaging of the nebula. Our most detailed comparison for model images was the $12.5 \mu \mathrm{m}$ MIRAC image. Measurement of the angular size of the nebula in the mid-IR images provides a fairly reliable input value for $r_{\min }$. Our modeling does not extend to the level of detail seen in the $2.2 \mu \mathrm{m}$ NICMOS image, as the smaller structural details are probably results of irregular mass loss or shell fragmentation, which we cannot reproduce. For all previous modeling pursued with 2-Dust, the value of $r_{\max }$ has been somewhat speculative and usually assumed to be factors of 100 more than the inner radius. However, for the case of HD 168625 the ISO $55 \mu \mathrm{m}$ image provides a critical constraint on the maximum extent of the dust shell. Since the nebula is unresolved in the ISO image, a model image must likewise be unresolved when convolved with a Gaussian distribution of size equal to the PSF of the ISOPHOT data. This size is similar to the mid-infrared shell size, indicating that the thickness of the shell is small.

Mid-IR spectra taken by Skinner (1997) indicate the presence of silicates in the dust shell of HD 168625. Moreover, a detailed study of the dust shells surrounding two other LBVs, Wra 751 and AG Car, shows that amorphous silicates dominate the dust composition (Voors et al. 2000). Hence, for the model dust distribution, we used optical constants determined for amorphous silicates $\left(\mathrm{MgFeSiO}_{4}\right.$; Dorschner et al. 1995). 2-Dust does not account for transiently heated very small dust grains or PAH emission, which are evident in our NICMOS image and in the mid-IR spectra of Skinner (1997) and the IRAS LRS spectrum. Therefore, we did not attempt to fit the SED in the 8-15 $\mu \mathrm{m}$ wavelength region.

Most estimates of the effective temperature of HD 168625 have been in the range 12,000-15,000 K, and of course, as with all LBVs the temperature varies over a range of a few thousand degrees. Since our SED contains data points taken by many observers over several decades, we cannot hope to model HD 168625 at any particular point in time. Similarly, the model value for the stellar radius of HD 168625 was adjusted to roughly match the observed SED and cannot be treated as a certain, constant value. Extra reddening above that of the circumstellar shell is required to redden the star to the observed SED. We use an estimate of the interstellar medium (ISM) extinction from Neckel \& Klare (1980), which at a distance of $2.8 \mathrm{kpc}$ is $A_{V} \simeq 4.6$.

\subsection{Model Results}

Approximately 160 models were run to obtain a best fit to the SED and data images. The best-fit SED, including ISM reddening using the method of Mathis (1990), is shown in Figure 5, and model images at 2.2, 12.5, and $20.6 \mu \mathrm{m}$ are shown in Figure 6, along with the corresponding data images from this work and Meixner et al. (1999). Input and 


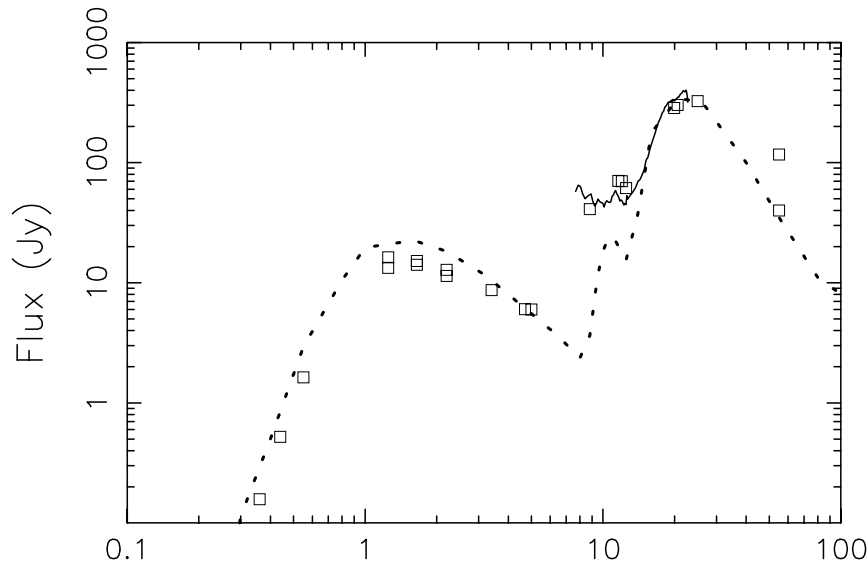

Wavelength $(\mu \mathrm{m})$

FIG. 5.-Observed and model SEDs of HD 168625. The reddened model SED is shown as a dashed line. The solid line is the IRAS LRS (Volk \& Cohen 1989). Squares are photometry data points compiled from Robberto \& Herbst (1998), Meixner et al. (1999), Ueta et al. (2003), this work's ISO data, the IRAS Point Source Catalog, and the SIMBAD database.

derived stellar and dust shell parameters are given in Tables 1 and 2. The values given in the tables are those of the final model, and the uncertainties are estimated from the range of parameters used in the modeling process.
The model SED fits the data well in the region of the spectrum above $20 \mu \mathrm{m}$, although it fits the $\sim 10-20 \mu \mathrm{m}$ less well for reasons explained above. The model flux at $55 \mu \mathrm{m}$ is consistent with our value of $\simeq 40 \mathrm{Jy}$ from the $I S O$ data.

Model images at 12.5, 20.6, 55, and $2.2 \mu \mathrm{m}$ were compared with the observations. By design, the 12.5 and 20.6 $\mu \mathrm{m}$ model images match the data well except for the gap in the southern rim of the $20.6 \mu \mathrm{m}$ emission, which may indicate the absence of large dust grains as discussed in $\S 4$. The inner radius of the dust shell is well constrained by these mid-IR images to a value of $0.11 \pm 0.01 \mathrm{pc}$. The $55 \mu \mathrm{m}$ model image (not shown) is unresolved when it is convolved with the $I S O$ beam and thus matches the data (Fig. 2) well. The $I S O$ image provides the best constraint on a value for $r_{\max }$ of $5 r_{\min }$, or $r_{\max } \simeq 0.6 \mathrm{pc}$. The model image at $2.2 \mu \mathrm{m}$ features a peak dust emission value $\left(10^{-3} \mathrm{Jy} \operatorname{arcsec}^{-2}\right)$ well below that of our NICMOS image $\left(9 \times 10^{-3} \mathrm{Jy} \operatorname{arcsec}^{-2}\right)$. Only emission from scattered starlight is present in our model at this wavelength. The difference between the images supports our suggestion that the dust shell includes small, transiently heated dust grains that are not at thermal equilibrium and are not accounted for in the model.

This model produces a dust temperature of $\simeq 130 \mathrm{~K}$ at $r_{\min }$. Given the low range of temperatures $(\sim 120 \mathrm{~K})$ in the dust shell as shown in Fig. $3 c$, this is in good agreement with blackbody dust temperatures of 135 and $113 \mathrm{~K}$ obtained by Robberto \& Herbst (1998) and Pasquali et al. (2002), respectively. The shell's dust opacity $\tau_{V} \sim 0.2$ at the equator

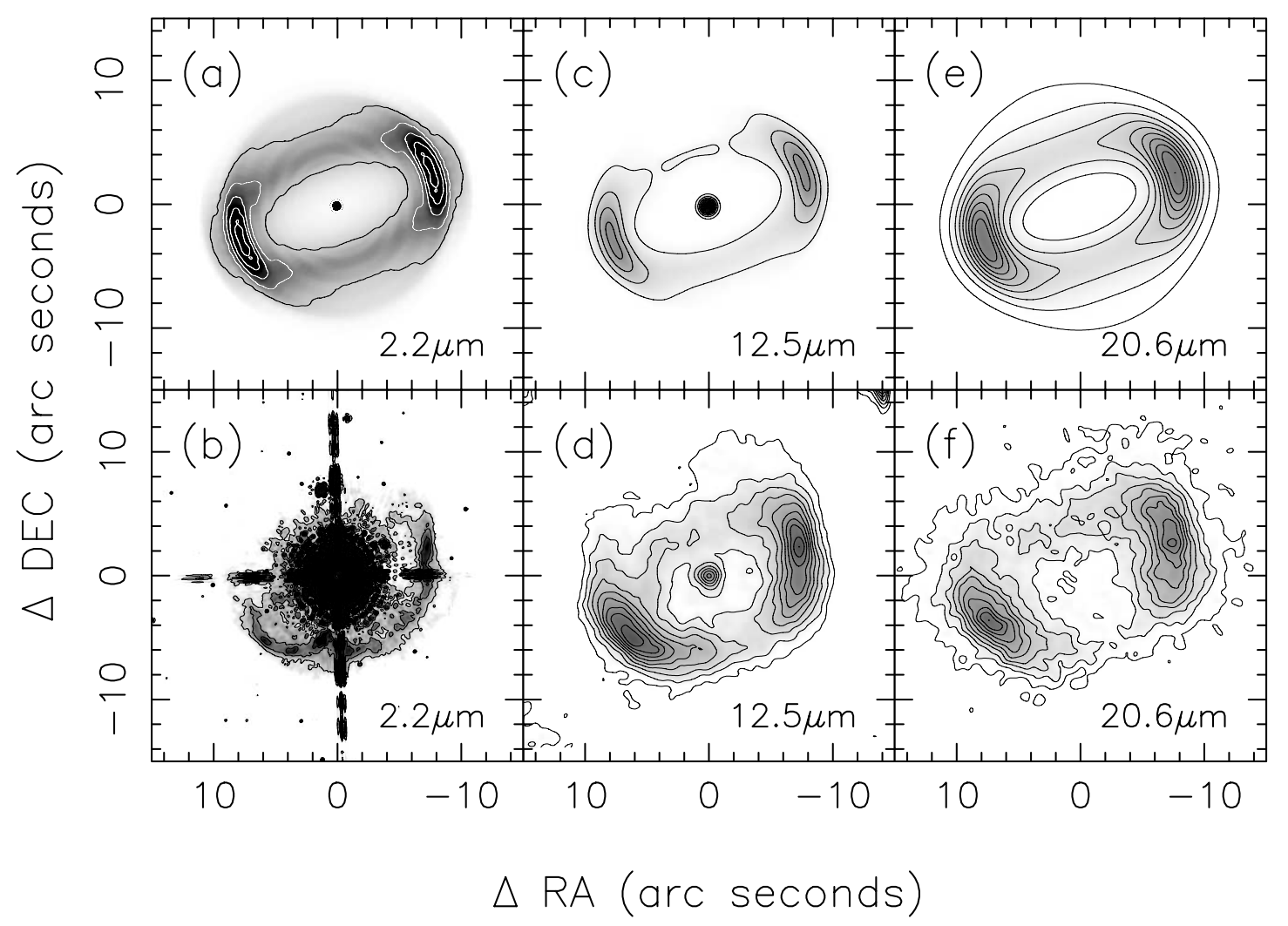

FIG. 6.- Model and data images of HD 168625 at $(a, b) 2.2 \mu \mathrm{m},(c, d) 12.5 \mu \mathrm{m}$, and $(e, f) 20.6 \mu \mathrm{m}$, with north up and east to the left. The models are shown with the disk inclined at an angle of $60^{\circ}$ with respect to the sky and have not been reddened to match the data images. In $(a)$ and $(b)$, contours are spaced at $20 \%$ of the peak dust shell intensity $\left(10^{-3} \mathrm{Jy} \mathrm{arcsec}-2\right.$ in $[a]$ and $9 \times 10^{-3} \mathrm{Jy} \mathrm{arcsec}^{-2}$ in $\left.[b]\right)$. The great difference in dust shell intensity indicates that the $2.2 \mu \mathrm{m}$ emission arises from transiently heated small grains, which are not included in our model. In the other images contours are spaced at intervals of $10 \%$ of the peak image intensity ( $0.4 \mathrm{Jy} \mathrm{arcsec}^{-2}$ in $[c]$ and $[d], 2.9 \mathrm{Jy} \mathrm{arcsec}^{-2}$ in $[e]$, and $3.2 \mathrm{Jy} \operatorname{arcsec}^{-2}$ in $\left.[f]\right)$. Model images have been convolved with a Gaussian with FWHM equal to that of the PSF of the corresponding data image. 
TABLE 1

InPUT and Derived Parameters: Stellar

\begin{tabular}{|c|c|c|}
\hline Parameter & Value & Reference \\
\hline$L_{*} \propto d \ldots \ldots \ldots \ldots \ldots \ldots$ & $8.6 \times 10^{5} L_{\odot}$ & \\
\hline$T_{\mathrm{eff}}$ & $14000 \mathrm{~K}$ & \\
\hline$R_{*} \propto d \ldots \ldots \ldots \ldots \ldots \ldots \ldots \ldots \ldots \ldots$ & $150 R_{\odot}$ & \\
\hline d & $2.8 \mathrm{kpc}$ & 1 \\
\hline ISM $A_{V} \ldots \ldots \ldots \ldots \ldots$ & 4.6 & 2 \\
\hline
\end{tabular}

ReferenCeS.-(1) Pasquali et al. 2002; (2) Neckel \& Klare 1980

of the torus. This equatorial dust opacity is comparable to the dust opacity estimated by Robberto \& Herbst (1998) for a spherical shell around HD 168625. The grain size distribution suggests that grain sizes between $0.005 \mu \mathrm{m}\left(a_{\min }\right)$ and $1.0 \mu \mathrm{m}\left(a_{\max }\right)$ exist in this circumstellar environment, consistent with models of other LBVs, e.g., $\eta$ Car (Mitchell \& Robinson 1986).

A range of model geometries were tried, ranging from a nearly spherical dust distribution to a basic torus. The most simple geometries were not sufficient to model the morphology of HD 168625, the determined shape of which is described by the parameters given in Table 3. It is largely disklike, with an equator-to-pole density ratio of $\sim 31$, but with an elliptical midshell that is controlled primarily by the input parameter $C$ in equation (1). Our calculated equatorto-pole density ratio is a factor of 3 higher than the semianalytic estimate by Nota et al. (1996). The model SED and images were calculated for multiple inclination angles, where the inclination angle is $90^{\circ}$ when the disk is viewed edge-on and $0^{\circ}$ when viewed pole-on. An inclination of $60^{\circ}$ was found to adequately reproduce observations and is used for the results presented here, although angles within $10^{\circ}$ $15^{\circ}$ of this produced quantitatively and qualitatively acceptable results.

This geometry of the shell reflects our qualitative picture of HD 168625 described in $\S 4$. Our model gives

TABLE 2

InPut and Derived Parameters: Dust Shell

\begin{tabular}{|c|c|c|}
\hline Parameter & Value & Reference \\
\hline \multicolumn{3}{|c|}{ Input } \\
\hline$v_{\exp } \ldots \ldots \ldots \ldots \ldots \ldots \ldots \ldots \ldots$ & $19 \mathrm{~km} \mathrm{~s}^{-1}$ & 1 \\
\hline$M_{\text {gas }} \propto d^{2}$ & $2.1 M_{\odot}$ & 1 \\
\hline 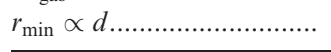 & $0.11 \pm 0.01 \mathrm{pc}$ & 2 \\
\hline \multicolumn{3}{|c|}{ Derived } \\
\hline 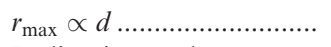 & $0.6 \pm 0.1 \mathrm{pc}$ & \\
\hline Inclination angle .................. & $60^{\circ} \pm 15^{\circ}$ & \\
\hline 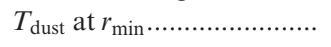 & $130 \mathrm{~K}$ & \\
\hline 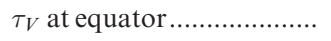 & $0.2 \pm 0.1$ & \\
\hline$a_{\min }$ & $0.005 \mu \mathrm{m}$ & \\
\hline$a_{\max }$ & $1.0 \mu \mathrm{m}$ & \\
\hline$t_{\exp } \propto d$ & $5700 \mathrm{yr}$ & \\
\hline 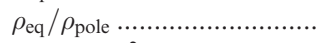 & 31 & \\
\hline$M_{\text {dust }} \propto d^{2} \ldots \ldots \ldots \ldots \ldots \ldots$ & $(2.5 \pm 0.1) \times 10^{-3} M_{\odot}$ & \\
\hline$\dot{M}_{\text {dust }} \propto d^{2} \ldots \ldots \ldots \ldots \ldots \ldots \ldots \ldots$ & $(2.2 \pm 0.1) \times 10^{-7} M_{\odot} \mathrm{yr}^{-1}$ & \\
\hline$M_{\text {gas }} / M_{\text {dust }} \ldots \ldots \ldots \ldots \ldots \ldots \ldots$ & 840 & \\
\hline$\dot{M}_{\text {shell }} \propto d^{2} \ldots \ldots \ldots \ldots \ldots \ldots$ & $(1.9 \pm 0.1) \times 10^{-4} M_{\odot} \mathrm{yr}^{-1}$ & \\
\hline
\end{tabular}

References.-(1) Pasquali et al. 2002; (2) this work.
TABLE 3

Density Function Parameters FOR BEST-FIT MODEL

\begin{tabular}{|c|c|}
\hline Parameter & Value \\
\hline 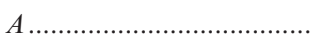 & 30 \\
\hline В & 2 \\
\hline С & 4 \\
\hline$D$ & 6 \\
\hline 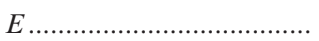 & 3 \\
\hline$F$ & 4 \\
\hline 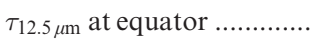 & 0.0012 \\
\hline
\end{tabular}

dust mass and dust mass-loss rate values of $2.5 \times 10^{-3} M_{\odot}$ and $2.2 \times 10^{-7} M_{\odot} \mathrm{yr}^{-1}$, respectively. Our result for the dust mass is a factor of 10 higher than the estimate by Hutsemékers $(1994,1997)$, who used 25 and $60 \mu \mathrm{m} I R A S$ fluxes; the latter we have shown is superseded by our ISOPHOT measurement. Nota et al. (1995) suggest that most LBV nebulae are density bounded; i.e., they are completely ionized. If we assume complete ionization and adopt the ionized gas mass of $2.1 M_{\odot}$ determined by Pasquali et al. (2002), then we find a rather high gas-to-dust mass ratio of 840 , which is a factor of $\sim 4$ above the current ISM value. This ratio then gives a total (gas plus dust) shell mass-loss rate of $1.9 \times 10^{-4} M_{\odot}$ and a total mass of $2.1 M_{\odot}$, which is well within the range $\left(\sim 2-4 \quad M_{\odot}\right)$ typical for LBV nebulae (Nota et al. 1995). Assuming that this shell is undisturbed from the mass-loss episode that created it, we can estimate timescales from the sizes of the shell. Dividing our value for $r_{\min }=0.11 \mathrm{pc}$ by the nebular expansion velocity of $19 \mathrm{~km} \mathrm{~s}^{-1}$ determined by Pasquali et al. (2002) gives an expansion time of $\simeq 5700 \mathrm{yr}$. Based on our $r_{\max }=0.6$ $\mathrm{pc}$, the mass loss endured for $\simeq 23,000 \mathrm{yr}$. The brevity of this mass-loss episode does not support the suggestion by Robberto \& Herbst (1998) that HD 168625's shell is a red supergiant wind swept up by the current LBV fast wind. The small outer radius, defined by the ISO $55 \mu \mathrm{m}$ image, demonstrates that there is no large neutral halo as Robberto \& Herbst (1998) suggested. Instead, our model results and data suggest that HD 168625 lost this shell in a torus-shaped "outburst," as has been suggested for all LBVs by Hutsemékers (1994). This toroidal morphology may have been formed by stellar rotation (Dwarkadas \& Owocki 2002). The current fast wind of the LBV has probably interacted with this torus, creating an elliptical bubble perpendicular to the plane of the torus, which is consistent with a unified model for LBV nebulae proposed by Nota et al. (1995).

\section{DISCUSSION}

The infrared images of HD 168625's nebula show similar structure to the nebula of the newly discovered LBV, RAFGL 2298, which is obscured completely in the optical by interstellar dust (Ueta et al. 2001a), and to the LBVs Wra 751 and AG Car (Voors et al. 2000). In all four cases, the nebulae are detached from the central star because they were ejected long ago, and there are limb-brightened peaks indicating optically thin, inclined dust tori. The optical nebula for HD 168625 is spatially coincident with its infrared emission. Such a coincidence is also observed in the 
TABLE 4

LBV SHELL PARAMETERS

\begin{tabular}{lcrc}
\hline \hline \multicolumn{1}{c}{ Object } & $\begin{array}{c}\text { Mass-Loss Rate } \\
\left(M_{\odot} \mathrm{yr}^{-1}\right)\end{array}$ & Equator/Pole Density Ratio & References \\
\hline \multicolumn{2}{c}{ LBVs } \\
\hline Car & 0.1 & 100 & 1,2 \\
HD 168625 & $1.9 \times 10^{-4}$ & 31 & 3,4 \\
\hline \multicolumn{2}{c}{ PPNs } & 9 & 5 \\
\hline HD 161796 & $1.2 \times 10^{-4}$ & 160 & 5 \\
IRAS 17150-3224 & $8.5 \times 10^{-3}$ & & \\
\hline
\end{tabular}

References.- (1) Nota et al. 1995; (2) Frank, Balick, \& Davidson 1995; (3) this work; (4) Pasquali et al. 2002; (5) Meixner et al. 2002.

most famous LBV, $\eta$ Car (Smith et al. 2002a); however, the shapes of $\eta \mathrm{Car}$ and HD 168625 differ markedly. HD 168625 is much less optically thick and equatorially enhanced than $\eta$ Car, which has an hourglass-shaped bipolar morphology. While some of these differences may be due to the fact that $\eta$ Car is much younger, at $\sim 150 \mathrm{yr}$ (Nota et al. 1995), and its ejecta are much closer, the quantitative analyses of these two sources suggest otherwise.

Little work has been done to quantify the geometry of mass loss in LBVs. Frank, Balick, \& Davidson (1995) have derived some numbers for this geometry for $\eta$ Car based on hydrodynamic modeling; we have derived numbers for HD 168625. In Table 4, we compare two values from these efforts, the mass-loss rate $(M)$ and the equator-to-pole mass-loss ratio. $\eta$ Car has a higher equator-to-pole ratio (100) and a substantially higher $\dot{M} \sim 0.1 M_{\odot} \mathrm{yr}^{-1}$ than HD 168625. These two points suggest that perhaps higher massloss rates create or require higher equator-to-pole mass-loss ratios. A similar tendency is found in intermediate-mass objects, known as PPNs, which are objects in transition from the asymptotic giant branch (AGB) to the planetary nebula phase. Meixner et al. (2002) performed radiative transfer modeling for examples from two morphological types of PPNs, which they labeled as SOLE (star-obvious low-level elongated) and DUPLEX (dust-prominent longitudinally extended) as first conceived by Ueta, Meixner, \& Bobrowsky (2000). One type, HD 161796 (a SOLE PPN), had a visible star, much like HD 168625, and was found to have a lower optical depth along the equator $\left(\tau_{V}=1.4\right)$, a lower apparent mass-loss rate $\left(1.2 \times 10^{-4} M_{\odot} \mathrm{yr}^{-1}\right)$, and a lower equator-to-pole mass-loss ratio (9). The second type, IRAS 17150-3224 (a DUPLEX PPN), had its star enshrouded by an optically thick torus, much like $\eta$ Car, and was found to have a higher optical depth $\left(\tau_{V}=37\right)$, a higher apparent mass-loss rate $\left(85 \times 10^{-4} M_{\odot} \mathrm{yr}^{-1}\right)$, and a higher equator-to-pole mass-loss ratio (160). Meixner et al. (2002) suggested that DUPLEX PPNs originated from higher mass progenitors that not only had higher mass-loss rates but also lost the mass in a more equatorially preferred direction compared with SOLE PPNs. Based on the similarities of the LBVs and PPNs, we suggest that perhaps higher mass progenitors produce $\eta$ Car-like LBVs while lower mass progenitors produce HD 168625-like LBVs.

The formation of LBVs may in fact follow the formation of PPNs and planetary nebulae even more closely. In the formation of planetary nebulae, an axisymmetric mass loss on the AGB creates an equatorial density enhancement in the ejected shell. This axisymmetric mass loss may arise from the interaction of the AGB star with a binary companion (Soker 2002; Soker \& Rappaport 2001). The interaction of a fast wind with this axisymmetric mass loss sculpts the shell, creating bubbles in the direction perpendicular to the equatorial torus (Balick \& Frank 2002; Frank \& Mellema 1994; Mellema 1995). Indeed, such a scenario has been espoused for LBVs by, e.g., Nota et al. (1995). Along these lines, it is interesting to compare the morphology of mass-loss shells for two high-mass evolved stars that may be potential precursors to LBVs. The $\mathrm{M}$ supergiant $\alpha$ Orionis has a relatively symmetric mass-loss shell that appears asymmetric because of a hot spot in its photosphere (Skinner et al. 1997). On the other hand, the post-main-sequence, contact binary system RY Scuti, which has two O stars, has recently ejected a ring nebula (Smith, Gehrz, \& Goss 2001; Gehrz et al. 2001; Smith et al. 2002b). This contrast in morphologies suggests that binaries may indeed influence the shaping of the high-mass star progenitors of LBVs as has been suggested for $\eta$ Car by Soker (2001).

While there are some similarities between LBVs and PPNs, there is an important difference. The mass loss that produces LBVs has a shorter duration than the mass loss that produces PPNs. The far-IR imaging provides the best evidence for the duration of the mass loss because it images the cooler dust farther from the star. Our $55 \mu \mathrm{m}$ image of HD 168625 suggests that the outer shell is not more extended than 5 times the inner radius. However, for the Egg Nebula, a well-studied PPN, the outer shell is a few hundred times larger than the inner radius as revealed by $180 \mu \mathrm{m}$ ISOPHOT data (Speck, Meixner, \& Knapp 2000). Thus, the mass-loss shells of LBVs appear to be more compressed than for PPNs, suggesting that the mass loss occurred in a more short-lived $\left(<10^{4} \mathrm{yr}\right)$, perhaps even eruptive, phase compared with PPNs $\left(\sim 10^{5} \mathrm{yr}\right)$.

\section{CONCLUSIONS}

Our $2.218 \mu \mathrm{m}$ NICMOS image and our $55 \mu \mathrm{m}$ ISOPHOT image of HD 168625 are spatially coincident with previously obtained ground-based mid-IR images. Our dust optical depth and color temperature maps, derived from mid-IR images, show evidence for the limb-brightened peaks of an equatorial torus and for transiently heated, very small dust grains. These results indicate that all the dust resides in a geometrically thin, axisymmetric shell. The NICMOS image, which traces the smallest dust grains, reveals that the shell is 
fragmented on small scales. An overall trend in the dust emission location with wavelength is observed and interpreted as a difference in either the dust grain size distribution or gasto-dust mass ratio with location in the nebula. Our detailed radiative transfer model using 2-Dust reveals that HD 168625 lost $(2.5 \pm 0.1) \times 10^{-3} M_{\odot}$ of dust in a massive stellar wind, with $\dot{M}_{\text {shell }}=(1.9 \pm 0.1) \times 10^{-4} M_{\odot} \mathrm{yr}^{-1}$, that ended $\sim 5700 \mathrm{yr}$ ago. Dividing the gas mass estimates of Pasquali et al. (2002) by our dust mass gives a gas-to-dust mass ratio of 840 , which is a factor of 4 higher than current estimates for the ISM. Comparison of our results with observations of other LBVs and their lower mass counterparts, PPNs, shows a broad similarity in bipolar morphology and supports suggestions that the physical characteristics that may shape PPNs (binarity, progenitor mass, and interacting winds) may also shape LBV nebulae.
This research has made use of the SIMBAD database, operated at CDS, Strasbourg, France. The ISOPHOT data presented in this paper were reduced using PIA, which is a joint development by the ESA Astrophysics Division and the ISOPHOT Consortium with the collaboration of the Infrared Processing and Analysis Center (IPAC). Contributing ISOPHOT Consortium institutes are DIAS, RAL, AIP, MPIK, and MPIA.

M. Meixner, T. B. O'Hara, A. K. Speck, and T. Ueta were partially supported by NSF CAREER grant AST 97-33697. A. K. Speck was also supported by NASA JPL 961504 and NASA STI 7898.02-96A. M. Bobrowsky was supported by NASA through grant HST-GO-07378.02-A from the Space Telescope Science Institute, which is operated by the Association of Universities for Research in Astronomy, Inc., under NASA contract NAS5-26555.
Balick, B., \& Frank, A. 2002, ARA\&A, 40, 439

Chentsov, E. L.. \& Luud, L. 1989, Astrofizika, 31, 5

Clampin, M., Nota, A., Golimowski, D., Leitherer, C., \& Durrance, S. 1993, ApJ, 410, L35

Clampin, M., Schulte-Ladbeck, R., Nota, A., Robberto, M., Paresce, F., \& Clayton, G. C. 1995, AJ, 110, 251

Conti, P. S. 1984, in Observational Tests of the Stellar Evolution Theory, ed. A. Maeder \& A. Renzini (Dordrecht: Reidel), 233

Davidson, K. 1987, in Instabilities in Luminous Early-Type Stars, ed.

H. J. G. L. M. Lamers \& C. W. H. de Loore (Dordrecht: Reidel), 127

Dayal, A., Hoffmann, W. F., Bieging, J. H., Hora, J. L., Deutsch, L. K., \& Fazio, G. G. 1998, ApJ, 492, 603

Dickinson, M. E., et al. 2002, in HST NICMOS Data Handbook, Version 5.0, ed. B. Mobasher (Baltimore: STScI), 3-5

Dorschner, J., Begemann, B., Henning, T., Jaeger, C., \& Mutschke, H. 1995, A\&A, 300, 503

Dwarkadas, V. V., \& Owocki, S. P. 2002, ApJ, 581, 1337

Frank, A., Balick, B., \& Davidson, K. 1995, ApJ, 441, L77

Frank, A., \& Mellema, G. 1994, ApJ, 430, 800

Gabriel, C., \& Hur, M. 2000, in ISO Beyond Point Sources: Studies of Extended Infrared Emission, ed. R. J. Laureijs, K. Leech, \& M. F. Kessler (ESA-SP 455; Noordwijk: ESA), 11

Gabriel, C., et al. 1997, in ASP Conf. Ser. 125, Astronomical Data Analysis Software and Systems VI, ed. G. Hunt \& H. E. Payne (San Francisco: ASP), 108

Gehrz, R. D., Smith, N., Jones, B., Puetter, R., \& Yahil, A. 2001, ApJ, 559, 395

Hanson, M. M., Conti, P. S., \& Rieke, M. J. 1996, ApJS, 107, 281

Humphreys, R. M., \& Davidson, K. 1994, PASP, 106, 1025

Hutsemékers, D. 1994, A\&A, 281, L81

. 1997, in ASP Conf. Ser. 120, Luminous Blue Variables: Massive Stars in Transition, ed. A. Nota \& H. J. G. L. M. Lamers (San Franciso: ASP), 316

Hutsemékers, D., Van Drom, E., Gosset, E., \& Melnick, J. 1994, A\&A, 290,906

Kessler, M. F., et al. 1996, A\&A, 315, L27

Leitherer, C., Schmutz, W., Abbott, D. C., Hamann, W.-R., \&

Wesselowski, U. 1989, ApJ, 346, 919

Lemke, D., et al. 1996, A\&A, 315, L64

Mathis, J. S. 1990, ARA\&A, 28, 37

Mathis, J. S., Rumpl, W., \& Nordsieck, K. H. 1977, ApJ, 217, 425

\section{EFERENCES}

Meixner, M., Ueta, T., Bobrowsky, M., \& Speck, A. 2002, ApJ, 571, 936

Meixner, M., et al. 1999, ApJS, 122, 221

Mellema, G. 1995, MNRAS, 277, 173

Mitchell, R. M., \& Robinson, G. 1986, MNRAS, 222, 347

Neckel, T. H., \& Klare, G. 1980, A\&AS, 42, 251

Nota, A., Leitherer, C., Clampin, M., Greenfield, P., \& Golimowski, D. A. G. 1992, ApJ, 398, 621

Nota, A., Livio, M., Clampin, M., \& Schulte-Ladbeck, R. 1995, ApJ, 448, 788

Nota, A., Pasquali, A., Clampin, M., Pollacco, D., Scuderi, S., \& Livio, M. 1996, ApJ, 473, 946

Pasquali, A., Nota, A., Smith, L. J., Akiyama, S., Messineo, M., \& Clampin, M. 2002, AJ, 124, 1625

Robberto, M., \& Herbst, T. M. 1998, ApJ, 498, 400

Schulz, B., \& Peschke, S., eds. 2002, Proc. ISOPHOT Workshop on P32 Oversampled Mapping (ESA-SP; Noordwijk: ESA), 93

Skinner, C. J. 1997, in ASP Conf. Ser. 120, Luminous Blue Variables: Massive Stars in Transition, ed. A. Nota \& H. J. G. L. M. Lamers (San Franciso: ASP), 322

Skinner, C. J., Dougherty, S. M., Meixner, M., Bode, M. F., Davis, R. J., Drake, S. A., Arens, J. F., \& Jernigan, J. G. 1997, MNRAS, 288, 295

Smith, N., Gehrz, R. D., \& Goss, W. M. 2001, AJ, 122, 2700

Smith, N., Gehrz, R. D., Hinz, P. M., Hoffmann, W. F., Mamajek, E. E., Meyer, M. R., \& Hora, J. L. 2002a, ApJ, 567, L77

Smith, N., Gehrz, R. D., Stahl, O., Balick, B., \& Kaufer, A. 2002b, ApJ, 578,464

Soker, N. 2001, MNRAS, 325, 584

. 2002, MNRAS, 330, 481

Soker, N., \& Rappaport, S. 2001, ApJ, 557, 256

Speck, A. K., Meixner, M., \& Knapp, G. R. 2000, ApJ, 545, L145

Ueta, T., \& Meixner, M. 2003, ApJ, 586, 1338

Ueta, T., Meixner, M., \& Bobrowsky, M. 2000, ApJ, 528, 861

Ueta, T., Meixner, M., Dayal, A., Deutsch, L. K., Fazio, G. G., Hora, J. L., \& Hoffman, W. F. 2001a, ApJ, 548, 1020

Ueta, T., Meixner, M., Moser, D. E., Pyzowski, L. A., \& Davis, J. S. 2003 , AJ, 125, 2227

Ueta, T., et al. 2001b, ApJ, 557, 831

van Genderen, A. M., et al. 1992, A\&A, 264, 88

Volk, K., \& Cohen, M. 1989, AJ, 98, 1918

Voors, R. H. M., et al. 2000, A\&A, 356, 501 